\title{
The association between hearing impairment and neural envelope encoding at different ages
}

\author{
Tine Goossens*, Charlotte Vercammen, Jan Wouters, Astrid van Wieringen
}

KU Leuven - University of Leuven, Department of Neurosciences, Research Group Experimental ORL (ExpORL), Herestraat 49 bus 721, 3000 Leuven, Belgium

\section{${ }^{*}$ Corresponding author:}

e-mail: tine.goossens@kuleuven.be postal address: Tine Goossens, KU Leuven - University of Leuven, Department of Neurosciences, Research Group Experimental ORL, Herestraat 49 bus 721, 3000 Leuven, Belgium

\section{E-mail addresses:}

Tine Goossens: tine.goossens@kuleuven.be

Charlotte Vercammen: charlotte.vercammen@kuleuven.be

Jan Wouters:

jan.wouters@med.kuleuven.be

Astrid van Wieringen:

astrid.vanwieringen@kuleuven.be 


\section{Abstract}

Hearing impairment goes with speech perception difficulties, presumably not only due to poor hearing sensitivity but also to altered central auditory processing. Critical herein is temporal processing of the speech envelope, mediated by synchronization of neural activity to the envelope modulations. It has been suggested that hearing impairment is associated with enhanced sensitivity to envelope modulations which, in turn, relates to poorer speech perception. To verify this hypothesis, we performed a comparative electrophysiological study in hearing-impaired $(\mathrm{HI})$ and normal-hearing $(\mathrm{NH})$ human listeners of three age groups, investigating neural envelope encoding. HI young and middleaged adults show enhanced neural synchronization to envelope modulations relative to $\mathrm{NH}$ controls, particularly when stimulus audibility is corrected for. At an older age, the degree of neural synchronization is similar in $\mathrm{HI}$ and $\mathrm{NH}$ persons, yet $\mathrm{HI}$ persons show a synchronization asymmetry towards the right hemisphere. This study demonstrates that hearing impairment is characterized by changes in the neural encoding of envelope modulations, the nature of which varies with age.

\section{Keywords}

hearing impairment | envelope modulations | neural synchronization | age | audibility 


\section{Introduction ${ }^{1}$}

Good speech perception skills are vital for communication and social integration. Hearing impairment, however, greatly degrades speech perception (e.g., Peters et al., 1998; Souza and Turner, 1994). Worldwide, 360 million persons (5.3\% of the world's population) suffer from moderate or greater degrees of hearing impairment and the prevalence of hearing impairment increases with advancing age (World Health Organization, 2012). According to the International Organization for Standardization (2017), $30 \%$ of persons aged 50 years or younger have hearing impairment, and at the age of 60 and 70 , this prevalence increases up to $50 \%$ and $90 \%$, respectively.

The poor hearing sensitivity (thresholds) of hearing-impaired $(\mathrm{HI})$ persons explains only a part of the inferior speech perception: when correcting for differences in hearing sensitivity between normalhearing $(\mathrm{NH})$ and $\mathrm{HI}$ persons, speech understanding is still worse in $\mathrm{HI}$ individuals (Eisenberg et al., 1995; George et al., 2006; Peters et al., 1998; Summers and Molis, 2004). It has been suggested that altered central auditory processing (supra-threshold), and temporal processing in particular, also accounts for the poor speech perception of $\mathrm{HI}$ individuals (Bernstein et al., 2013; Noordhoek et al., 2001). A central contribution to impaired speech perception is not unexpected, since numerous functional and structural changes emerge in the central auditory system throughout adult life, e.g., changes in neural inhibition (e.g., Caspary et al., 2008) and cortical thickness (e.g., Fjell et al., 2009). Moreover, evidence exists that such central changes are related to speech perception performance (Bilodeau-Mercure et al., 2015; Giroud et al., 2018).

Temporal processing plays a key role in speech perception since speech is characterized by modulations in its amplitude and spectrum across time (Rosen, 1992). Such acoustic modulations are encoded in the central auditory system through synchronized activity of neural oscillations: in response to an auditory input, neural oscillations in the human brain, i.e., delta $(<4 \mathrm{~Hz})$, theta (4-7 Hz), alpha (8$13 \mathrm{~Hz}$ ), beta $(14-30 \mathrm{~Hz})$, and gamma (> $30 \mathrm{~Hz})$ oscillations, synchronize to acoustic modulations corresponding to their characteristic frequency (Lopes da Silva, 2013; Peelle and Davis, 2012; Wang et al., 2005). It has been demonstrated that the entire central auditory system is responsive to any modulation frequency, yet cortical and subcortical auditory neurons show to be most responsive to,

Abbreviations: AM, amplitude modulation; ASSR, auditory steady-state response; BI, bilaterally; $\mathrm{HI}$, hearing-impaired; L, left ear; $\mathrm{LH}$, left hemisphere; LI, laterality index; MD, mean difference; $\mathrm{NH}$, normal-hearing; $\mathrm{R}$, right ear; $\mathrm{RH}$, right hemisphere; $\mathrm{SD}$, standard deviation; SNR, signal-to-noise ratio; SYM, symmetrical; TFS, temporal fine-structure 
respectively, low-frequency $(<30 \mathrm{~Hz})$ and higher-frequency modulations (Coffey et al., 2016; Giraud et al., 2000; Herdman et al., 2002; Joris et al, 2004). Another interesting feature of synchronized neural activity concerns a functional hemispheric asymmetry: neurons in the right hemisphere $(\mathrm{RH})$ preferentially synchronize to low-frequency acoustic modulations, while the left hemisphere (LH) mainly processes rapid acoustic modulations (Boemio et al., 2005; Poeppel, 2003).

Both behavioral and neuroimaging research have demonstrated that the speech envelope, i.e., the slowly fluctuating overall amplitude of continuous speech, is a crucial temporal speech feature. Drullman et al. (1994) showed that speech intelligibility is greatly degraded when the low-frequency speech envelope modulations are removed. Also, Shannon et al. (1995) observed nearly perfect intelligibility of speech when its spectral information was reduced, on the condition that the speech envelope was preserved. Likewise, electrophysiological studies show that neural synchronization to the speech envelope is a prerequisite for speech understanding (Ahissar et al., 2001; Doelling et al., 2014; Peelle and Davis, 2012). Given the importance of the speech envelope, it seems likely that speech perception difficulties in $\mathrm{HI}$ persons can be attributed, at least in part, to changes in temporal envelope processing. Note that we use the term "HI" throughout this paper to denote people who are diagnosed with peripheral hearing impairment as indicated by the audiogram, independent of altered central auditory processing.

Several behavioral studies have reported lower amplitude modulation (AM) detection thresholds, reflecting increased sensitivity to envelope modulations, in $\mathrm{HI}$ compared to $\mathrm{NH}$ listeners (Ernst and Moore, 2012; Moore et al., 1996; Sek et al., 2015; Wallaert et al., 2017). Other studies, however, found $\mathrm{AM}$ detection thresholds, and thus envelope sensitivity, to be similar among $\mathrm{NH}$ and $\mathrm{HI}$ individuals (Grose et al., 2016; Moore and Glasberg, 2001). This behavioral discrepancy can be attributed to stimulus audibility. In fact, increased sensitivity to envelope modulations in $\mathrm{HI}$ relative to $\mathrm{NH}$ persons, is only observed when stimulus audibility is corrected for, i.e., when adjusting the stimulus level for HI participants (Bacon and Gleitman, 1992; Schlittenlacher and Moore, 2016). Interestingly, when envelope modulations are perceptually enhanced, $\mathrm{NH}$ listeners show worse speech perception, especially when the speech is masked by background noise (Moore and Glasberg, 1993; Moore et al., 1995). This indicates that enhanced envelope processing is related to poorer speech perception, which may be explained by a processing imbalance between envelope and temporal fine-structure (TFS) cues, i.e., spectral speech features. In effect, processing of the TFS, 
next to the speech envelope is crucial for speech perception, especially when the speech is masked by background noise (Hopkins and Moore, 2009; Lorenzi et al., 2006). Moore and Glasberg (1993) and Moore et al. (1995) found envelope enhancement to be negatively correlated with speech perception performance for a $\mathrm{NH}$ population. Based on research demonstrating brain plasticity with hearing impairment (e.g., Neuman, 2005), caution should, however, be taken when generalizing these findings to the $\mathrm{HI}$ population.

In agreement with behavioral research, electrophysiological studies in animals have shown that hearing impairment coincides with increased neural synchronization to acoustic envelope modulations in subcortical structures, i.e., in the auditory nerve fibers (Henry et al., 2014; Kale and Heinz, 2010, 2012) and in the midbrain (Zhong et al., 2014). To date, only a few studies have investigated the association between hearing impairment and neural envelope encoding in the human brain. Millman et al. (2017) compared neural synchronization to envelope modulations in the auditory cortex between $\mathrm{HI}$ and NH similarly-aged persons ( $\sim 60$ years). To control for stimulus audibility, the stimulus was amplified for the HI participants. Increased synchronized neural activity was detected in the auditory cortex of $\mathrm{HI}$ relative to $\mathrm{NH}$ adults. Anderson et al. (2013) looked into brainstem neural synchronization in $\mathrm{NH}$ and $\mathrm{HI}$ adults who were on average 64 years old. The $\mathrm{HI}$ participants exhibited higher-thannormal neural envelope encoding, whether or not amplification was applied. Ananthakrishnan et al. (2016) did not find neural envelope encoding in the brainstem to be enhanced in $\mathrm{HI}$ persons between 21 and 71 years of age ( 50 years). Importantly, Ananthakrishnan et al. (2016) detected a similar increase in neural synchronization with stimulus level among $\mathrm{NH}$ and $\mathrm{HI}$ adults, and they therefore concluded that stimulus audibility should be taken into account when comparing neural envelope encoding between persons with different hearing sensitivity. This notion fits with the observed interplay between stimulus audibility and behavioral sensitivity to envelope modulations (Bacon and Gleitman, 1992; Schlittenlacher and Moore, 2016).

The observed negative correlation between envelope processing and speech perception performance for NH adults (Moore and Glasberg, 1993; Moore et al., 1995), has raised the idea that speech perception difficulties could be associated with enhanced neural envelope encoding. Recent studies support this idea and, even more, indicate that such neural-behavioral association applies to both $\mathrm{NH}$ and $\mathrm{HI}$ people. Millman et al. (2017) found that enhanced neural synchronization to acoustic envelope modulations recorded from the left auditory cortex, was predictive of worse speech perception for a 
middle-aged group ( 60 years) consisting of both $\mathrm{NH}$ and $\mathrm{Hl}$ listeners. Goossens et al. (2018) included young (20-30 years), middle-aged (50-60 years), and older (70-80 years) $\mathrm{NH}$ and $\mathrm{HI}$ adults and demonstrated that enhanced neural envelope encoding in the auditory cortex and in the brainstem, respectively, corresponded to poorer speech perception for $\mathrm{NH}$ and $\mathrm{HI}$ adults, irrespective of age.

As for the previously mentioned hemispheric asymmetry in neural envelope encoding, i.e., the differential temporal sensitivity of the $\mathrm{LH}$ and $\mathrm{RH}$, it has been documented that this is a prerequisite for good speech processing (for a review see Specht, 2014). Moreover, evidence exists that different hemispheric processing patterns relate to different speech perception abilities. Bellis et al. (2000) demonstrated that persons who performed well on a syllable discrimination task showed a LH processing asymmetry for the syllables, whereas a symmetrical processing pattern was observed for listeners who performed poorly on the discrimination task. Hämäläinen et al. (2012) evaluated neural envelope encoding in persons with good speech perception skills and in persons who were diagnosed with a speech processing disorder (dyslexia). The first group exhibited a $\mathrm{RH}$ asymmetry while no such hemispheric asymmetry was observed in the latter group. These studies - showing that speech perception correlates with functional hemispheric asymmetry - raise the question whether hearing impairment interferes with functional hemispheric asymmetry. This has thus far not been explored. Another unexplored aspect of neural envelope encoding concerns potential age-related variations. Neuroimaging studies in humans have been restricted to $\mathrm{HI}$ adults who are around 50-60 years of age. As aging is accompanied by changes in the degree and hemispheric asymmetry of neural envelope encoding (Anderson et al., 2012; Goossens et al., 2016), the association between hearing impairment and neural envelope encoding may very well vary with age. This remains to be investigated. In sum, an important lack of knowledge exists on neural envelope encoding in the $\mathrm{HI}$ human brain. Yet, such is needed for developing advanced intervention strategies for remediating impaired speech perception. To address this need, we performed a comparative study in $\mathrm{HI}$ and $\mathrm{NH}$ adults belonging to three age groups (young, middle-aged, older). While taking stimulus audibility into account, we investigated the degree as well as the hemispheric asymmetry of neural envelope encoding by measuring different neural responses of which the main sources are known to be located at different levels along the auditory pathway (i.e., from the brainstem up to the cortex). 
In line with previous electrophysiological research concerning $\mathrm{HI}$ subjects and reported correspondences between neural envelope encoding and speech perception, we hypothesized that $\mathrm{HI}$ human listeners would show enhanced neural envelope encoding compared to $\mathrm{NH}$ controls in both subcortical and cortical auditory regions. In the same vein, we presumed that $\mathrm{HI}$ and $\mathrm{NH}$ people would differ in functional hemispheric asymmetry. Moreover, hearing-related changes in neural envelope encoding were expected to be most evident when stimulus levels were adjusted for $\mathrm{HI}$ listeners, i.e., when correcting for stimulus audibility. Finally, as neural envelope encoding changes with age, we postulated that hearing impairment would differentially affect neural envelope encoding at different ages throughout adult life.

\section{Material and methods}

\subsection{Participants}

We recruited young (20-30 years), middle-aged (50-60 years), and older (70-80 years) adults who were either $\mathrm{NH}$ or $\mathrm{HI}$ (Table 1, Fig. 1). $\mathrm{HI}$ adults and their $\mathrm{NH}$ controls belonged to a narrow age cohort, spanning one decade.

\begin{tabular}{lllllll}
\hline & young & \multicolumn{3}{l}{ middle-aged } & older \\
\cline { 2 - 7 } & $\mathrm{NH}$ & $\mathrm{HI}$ & $\mathrm{NH}$ & $\mathrm{HI}$ & $\mathrm{NH}$ & $\mathrm{HI}$ \\
\cline { 2 - 7 } number & $20(11 / 9)$ & $10(6 / 4)$ & $20(10 / 10)$ & $14(10 / 4)$ & $14(10 / 4)$ & $13(8 / 5)$ \\
mean age \pm SD & $22 \pm 1$ & $27 \pm 5$ & $53 \pm 2$ & $57 \pm 2$ & $74 \pm 3$ & $78 \pm 3$ \\
\hline
\end{tabular}

Table 1. Number of persons (women/men) and mean age \pm standard deviation (SD), expressed in years, of the six participant groups.

$<<$ insert Fig. 1 about here $>>$

Only persons who showed symmetrical hearing, consistent with the criteria of the Audiogram Classification System (Margolis and Saly, 2008), were eligible for participation. A person was classified as $\mathrm{NH}$ when he/she had audiometric thresholds within normal limits, i.e., $\leq 25 \mathrm{~dB} H \mathrm{HL}$ (International Organization for Standardization, 1998), at all octave frequencies from $125 \mathrm{~Hz}$ up to 4 $\mathrm{kHz}$. HI participants had audiometric thresholds $\geq 35 \mathrm{~dB} \mathrm{HL}$ from $1 \mathrm{kHz}$ onwards. All hearing losses were sensorineural in nature (air-bone gaps $\leq 10 \mathrm{~dB} \mathrm{HL}$ ). The three age groups did differ in the etiology of their hearing impairment. In fact, young participants suffered from congenital hearing loss or hearing impairment following severe/chronic otitis, middle-aged adults had a family history of hearing loss, manifesting either before or after the age of 40 , and all older persons were diagnosed 
with age-related hearing loss, i.e., presbycusis. Except for two $\mathrm{HI}$ young participants, all HI participants were hearing aid users (bilateral).

Because cognitive deficiencies are known to alter neural processing (Bidelman et al., 2017), only individuals who showed no indication of cognitive impairment were included: all participants scored at or above the cut-off score of $26 / 30$ on the Montreal Cognitive Assessment (Nasreddine et al., 2005). All participants were Dutch native speakers, they were right-handed, as assessed by the Edinburgh Handedness Inventory (Oldfield, 1971), and they had no medical history of brain injury, neurological disorders, or tinnitus.

The study was approved by the Medical Ethical Committee of the University Hospitals and University of Leuven (approval number B322201214866). All participants gave their written informed consent.

\subsection{Auditory steady-state responses}

We measured auditory steady-state responses (ASSRs) to acoustic amplitude modulations, reflecting the degree of neural envelope encoding: larger ASSRs reflect a higher degree of neural synchronization (for a review on ASSRs see Rance, 2008). The stimuli and procedures for EEG recording, EEG processing, and ASSR evaluation were adopted from our previous study. An overview is given below. Detailed information can be retrieved from Goossens et al. (2016).

\subsubsection{Stimuli}

The acoustic stimulation set-up was controlled by a laptop running a custom made software platform. Octave bands of white noise centered at $1 \mathrm{kHz}$ were presented with ER-3A insert phones. The noise, generated in Matlab (The Mathworks Inc.), was 100\% sinusoidally amplitude modulated at 4, 20, 40, or $80 \mathrm{~Hz}$, representing theta, beta, low gamma, and high gamma frequencies, respectively (Fig. 2). ASSRs are composite responses, reflecting both cortical and subcortical neural activity, yet ASSRs to specific modulation frequencies originate primarily from specific auditory regions. Various neuroimaging studies indicate that the $4,20,40$, and $80 \mathrm{~Hz}$ ASSR included in the present study predominantly originate from the auditory cortex, thalamus, thalamus/upper brainstem, and brainstem, respectively (Herdman et al., 2002; Luke et al., 2017; Wang et al., 2012;). Every modulation frequency was presented to the left ear $(L)$, to the right ear $(R)$, and to both ears simultaneously, i.e., bilaterally (BI). We implemented different sides of stimulus presentations to verify whether functional hemispheric asymmetry depended on the side of stimulation, as indicated by previous research (Poelmans et al., 
2012). It was also a way to evaluate the robustness of hearing-related differences: a difference that was found for all three sides of stimulation, was considered as highly robust. For NH participants, the stimulus level was set to $70 \mathrm{~dB}$ SPL, which they rated as comfortably loud (graphic rating scale). To control for differences in stimulus audibility among $\mathrm{NH}$ and $\mathrm{HI}$ listeners - no hearing aids were used during EEG recording - the presentation level of the modulated noises for HI participants was individually determined. Every $\mathrm{HI}$ participant adjusted the stimulus level until he/she perceived it as comfortably loud, like the NH participants. In this way, the stimuli could be presented to all participants at equal loudness levels. The reason to use equal loudness levels to control for stimulus audibility instead of equal sensation levels is twofold. First, the sensation level of the $70 \mathrm{~dB}$ SPL stimulus equaled $65 \mathrm{~dB}$ SPL for $\mathrm{NH}$ participants. This would have implied presenting the stimuli at $\sim 108 \mathrm{~dB}$ SPL to HI participants, thereby exceeding their uncomfortable loudness level ( 103 dB SPL). Secondly, it has been demonstrated that the magnitude of the ASSR is highly correlated with the perceived loudness of the stimulus (Emara and Kolkaila, 2010; Ménard et al., 2008; Van Eeckhoutte et al., 2016). As such, using equal loudness levels is a good way to prevent changes in ASSR magnitude between $\mathrm{NH}$ and $\mathrm{HI}$ listeners to result from the stimulus presentation level. In addition to equal loudness levels, the acoustic modulations were also presented to the $\mathrm{HI}$ adults at equal SPL, i.e., 70 dB SPL. By using equal loudness levels as well as equal SPL, we could verify whether the relation between hearing impairment and neural envelope encoding depended on stimulus audibility. To reduce testing time, equal SPL conditions were restricted to $L$ stimulus presentation.

Stimulus level calibrations were performed with a Brüel and Kjær type 2260 sound level meter, a oneinch pressure-field microphone type 4144 and an artificial ear type 4152 (2 cc coupler).

$<<$ insert Fig. 2 about here $>>$

\subsubsection{EEG recording}

EEG recordings were made in a double-walled sound proof booth with Faraday cage. Participants lay down on a bed and watched a muted, subtitled movie. Each recording, i.e., for each stimulus condition (e.g., $4 \mathrm{~Hz} \mathrm{BI}$ ), lasted 300 seconds. EEG signals were recorded with the ActiveTwo system (BioSemi B.V.) including 64 active pin-type electrodes that were mounted in a head cap (Fig. 2). ActiView software was used to display the EEG signal on screen and store it on hard disk. 


\subsubsection{EEG processing}

Every EEG recording was segmented into epochs of $1.024 \mathrm{~s}$, each including an integer number of 4 , 20, 40, or $80 \mathrm{~Hz}$ modulation cycles (John and Picton, 2000). Artifact rejection was applied with BESA Research 5.3 software (BESA GmbH). The first 256 artifact-free epochs were imported into Matlab, referenced to $\mathrm{Cz}$, and chunked into four sweeps of 64 epochs. The sweeps were averaged in the time domain, thereby reducing the noise in the EEG signal (John et al., 1998). The average EEG time signal was converted into the frequency domain through a Fast Fourier Transform. In the resulting spectrum, the ASSR was represented by a spectral peak in the frequency bin corresponding to the presented acoustic modulation frequency (Fig. 2).

\subsubsection{ASSR evaluation}

The neural responses recorded by eight posterior electrode pairs were retained for further analyses

(Fig. 2). This electrode selection was adopted from our previous study showing that these electrodes were most sensitive to the neural responses under investigation (Goossens et al., 2016).

The magnitude of the ASSR, reflecting the degree of neural synchronization to the acoustic envelope modulations, can be investigated by the signal-to-noise ratio (SNR) (Eq. 1) or the amplitude (Eq. 2) of the neural response, calculated per hemisphere, i.e., across the eight electrodes selected in each hemisphere.

$\operatorname{SNR}(\mathrm{dB})=10 \times \log _{10} \frac{\sum_{\mathrm{i}=1}^{8} \frac{\mathrm{P}_{(\mathrm{S}+\mathrm{N})_{\mathrm{i}}}}{\mathrm{P}_{\mathrm{N}_{\mathrm{i}}}}}{8}$

amplitude $(\mathrm{dB})=20 \times \log _{10} \sqrt{\frac{\sum_{i=1}^{8} \mathrm{P}_{(\mathrm{S}+\mathrm{N})_{\mathrm{i}}}}{8}}$

$\mathrm{P}_{S_{+} \mathrm{N}}$ denotes the power of the Fourier component in the modulation frequency bin, including both the synchronized neural response $(\mathrm{S})$ and the induced EEG noise $(\mathrm{N})$. Induced EEG noise refers to random, non-synchronized neural activity during exposure to the acoustic modulations. We used the $F$ test to compare $P_{S+N}$ to $P_{N}$ (Dobie and Wilson, 1996). A significant ASSR was detected when the power in the modulation frequency bin was $\geq 3.03$ times higher than the power of the induced EEG noise, i.e., $S N R \geq 4.8 \mathrm{~dB}\left(10 \times \log _{10} 3.03\right)$. 
In addition to the degree of neural synchronization, we examined functional hemispheric asymmetry, i.e., the relative degree of neural synchronization across hemispheres. Hemispheric asymmetry was defined by three laterality categories: $\mathrm{LH}, \mathrm{RH}$, and SYM. For each modulation frequency $(4,20,40,80$ $\mathrm{Hz}$ ), per side of stimulus presentation ( $\mathrm{L}, \mathrm{R}, \mathrm{BI})$, every participant was classified into a laterality category. When a significant ASSR was only detected in the $\mathrm{LH}$ or $\mathrm{RH}$, the person was classified into these respective categories. If the participant exhibited a significant ASSR in both hemispheres, a laterality index (LI) was calculated (Eq. 3).

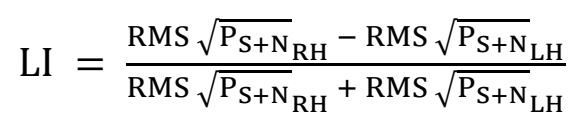

This LI represented the difference between the root mean square (RMS) response amplitude of the electrode selection in the $\mathrm{RH}$ and the $\mathrm{LH}$, normalized by the sum of the hemispheric response amplitudes. The $\mathrm{LI}$ could take on any value between -1 and +1 : a negative and positive $\mathrm{LI}$ denoted lateralization to the $\mathrm{LH}$ and $\mathrm{RH}$, respectively. If the absolute value of a person's $\mathrm{LI}$ exceeded the $25^{\text {th }}$ percentile of the median LI among all participants, the hemispheric asymmetry was considered to be strong and the subject was assigned a $\mathrm{LH}(\mathrm{LI}<0)$ or $\mathrm{RH}(\mathrm{LI}>0)$ asymmetry. LIs below the $25^{\text {th }}$ percentile were regarded as reflecting a negligible hemispheric asymmetry (SYM). The assignment of hemispheric asymmetry based on the LI, was in accordance with criteria used in other studies (Pujol et al., 1999).

\subsubsection{Statistical analyses}

All statistical analyses were performed with SPSS Statistics software (IBM Corp.). The association between hearing impairment and the degree of neural synchronization was investigated by comparing SNRs and response amplitudes between $\mathrm{HI}$ young, middle-aged, and older adults and their $\mathrm{NH}$ controls (Fig. 2). Shapiro-Wilk testing was conducted to assess the distributions of the SNR and response amplitude. When deviations from the normal distribution were detected, non-parametric analyses were performed to verify the parametric statistics. Since parametric and non-parametric statistics yielded the same results, we only report parametric testing. For each modulation frequency $(4,20,40,80 \mathrm{~Hz})$, we conducted a factorial mixed ANOVA with the SNR $(\mathrm{dB})$ or response amplitude (dB) as the dependent variable and hearing status $(\mathrm{NH}, \mathrm{HI})$ and age group (young, middle-aged, older) 
as the between-subject variables. For ASSRs to acoustic modulations presented at equal loudness levels, hemisphere $(\mathrm{LH}, \mathrm{RH})$ and side of stimulation $(\mathrm{L}, \mathrm{R}, \mathrm{BI})$ were the within-subject variables. For ASSRs to the modulations presented at equal SPL, hemisphere $(\mathrm{LH}, \mathrm{RH})$ was the sole within-subject, since only $L$ stimulus presentation was applied. All analyses were two-tailed $(\alpha=0.05)$. Mauchly's statistics determined whether or not a correction to the degrees of freedom had to be applied for examining within-subject effects. Post hoc comparisons were based on the conservative Bonferroni procedure.

As for the functional hemispheric asymmetry, we conducted binomial tests to identify significant hemispheric asymmetries, which, in turn, could be compared between $\mathrm{HI}$ and $\mathrm{NH}$ participants in order to examine the relationship between hearing impairment and hemispheric asymmetry. To correct for the multiple binomial testing, a corrected $\alpha$ level of 0.01 was applied. Per participant group, two binomial tests were performed for each stimulus condition (e.g., $4 \mathrm{~Hz} \mathrm{BI}$ ), yielding the exact probability of the observed number of participants in a specific laterality category under the null hypothesis that there was an equal number of participants in the two categories under investigation, e.g.., LH vs. RH and LH vs. SYM. A significant LH asymmetry was assigned to a participant group when significantly more subjects were classified into the category LH compared to both the category RH and SYM ( $P$ of both binomial tests $<0.01)$. Similar criteria applied to the assignment of a $\mathrm{RH}$ asymmetry or SYM processing. If no significant deviation from an equal distribution across laterality categories was detected, no significant hemispheric asymmetry $(\mathrm{LH}, \mathrm{RH})$ nor SYM processing was assigned to the participant group.

\section{Results}

\subsection{Degree of neural synchronization}

When presenting the acoustic envelope modulations at equal loudness levels, thereby correcting for stimulus audibility (Fig. 3), the following effects were observed. First, we found a significant interaction between hearing status, age group, and hemisphere for the degree of theta synchronization, reflected by the magnitude of the $4 \mathrm{~Hz} \operatorname{SNR}(F(2,83)=3.21, P=0.045)$. $\mathrm{HI}$ young adults showed higher $4 \mathrm{~Hz}$ SNRs than $\mathrm{NH}$ young listeners, particularly in the $\mathrm{LH}(4 \mathrm{~Hz} \mathrm{LH}$ : mean difference $(M D)=3.6 \mathrm{~dB}, P=$ $0.009 ; 4 \mathrm{~Hz} \mathrm{RH}: M D=2.8 \mathrm{~dB}, P=0.051)$. $\mathrm{HI}$ middle-aged participants also exhibited higher $4 \mathrm{~Hz}$ SNRs than their $\mathrm{NH}$ counterparts, and this was the case for both hemispheres $(4 \mathrm{~Hz} \mathrm{LH}: M D=3.2 \mathrm{~dB}$, $P=0.012 ; 4 \mathrm{~Hz} \mathrm{RH}: M D=3.0 \mathrm{~dB}, P=0.022) . \mathrm{HI}$ older individuals, on the contrary, showed similar 4 
$\mathrm{Hz}$ SNRs as NH older persons ( $4 \mathrm{~Hz} \mathrm{LH}: M D=0.9 \mathrm{~dB}, P=0.52 ; 4 \mathrm{~Hz} \mathrm{RH}: M D=0.4 \mathrm{~dB}, P=0.79)$. As for the 20 and $40 \mathrm{~Hz}$ SNRs, these did not differ significantly between $\mathrm{HI}$ and $\mathrm{NH}$ listeners for any of the three age groups (all $P>0.05$ ). For the $40 \mathrm{~Hz}$ SNR, there was a significant interaction between hearing status, age group, hemisphere, and side of stimulation $(F(4,166)=2.84, P=0.026)$. Post hoc comparisons demonstrated that $\mathrm{HI}$ and $\mathrm{NH}$ older participants differed in the relative degree of synchronization to $40 \mathrm{~Hz}$ modulations across hemispheres: $\mathrm{HI}$ older persons showed higher $40 \mathrm{~Hz}$ SNRs in the $\mathrm{RH}$ than in the $\mathrm{LH}$ for the three sides of stimulation $(\mathrm{L}, \mathrm{R}, \mathrm{BI}: M D=2.2 \mathrm{~dB}, 1.6 \mathrm{~dB}, 1.6 \mathrm{~dB}$, all $P<0.005)$, whereas $\mathrm{NH}$ older adults exhibited equally high $40 \mathrm{~Hz}$ SNRs in both hemispheres $(\mathrm{L}, \mathrm{R}$, $\mathrm{BI}: M D=0 \mathrm{~dB}, 0.5 \mathrm{~dB}, 0.2 \mathrm{~dB}$, all $P>0.3$ ). Finally, for the degree of high gamma synchronization, reflected by the $80 \mathrm{~Hz}$ SNR, we found a significant interaction between hearing status and age group $(F(2,84)=4.07, P=0.021)$. Young and middle-aged $\mathrm{HI}$ adults showed higher $80 \mathrm{~Hz} \mathrm{SNRs}$ than their $\mathrm{NH}$ controls (young: $M D=5.6 \mathrm{~dB}, P<0.001$; middle-aged: $M D=5.1 \mathrm{~dB}, P<0.001$ ), whereas similar $80 \mathrm{~Hz}$ SNRs were observed for $\mathrm{HI}$ and $\mathrm{NH}$ older listeners $(M D=0.5 \mathrm{~dB}, P=0.72)$

$<$ insert Fig. 3 about here $>>$

When using the response amplitude as a measure of the ASSR magnitude, statistical analyses identified the same hearing-related differences. The one exception to this concerned the $40 \mathrm{~Hz}$ ASSR. In contrast to the SNR, the amplitude did reveal hearing-related differences in the degree of synchronization to $40 \mathrm{~Hz}$ modulations for the young and middle-aged groups (Fig. 3). For the $40 \mathrm{~Hz}$ amplitude, we found a significant interaction between hearing status, age group, hemisphere, and side of stimulation $(F(3.45,143.19)=4.24, P=0.005)$. Compared to $\mathrm{NH}$ young adults, $\mathrm{HI}$ young participants showed higher $40 \mathrm{~Hz}$ amplitudes in the $\mathrm{LH}$ when the $40 \mathrm{~Hz}$ modulations were presented to the left ear $(\mathrm{L})(M D=3.1 \mathrm{~dB}, P=0.032)$ and in the $\mathrm{RH}$ in the case of right ear $(\mathrm{R})$ stimulus presentation $(M D=2.7$ $\mathrm{dB}, P=0.038)$. $\mathrm{HI}$ middle-aged adults showed higher $40 \mathrm{~Hz}$ amplitudes than $\mathrm{NH}$ middle-aged adults in both hemispheres for unilateral stimulus presentations $(\mathrm{L} L H, R \mathrm{LH}, \mathrm{L} R H, \mathrm{RH}: M D=4.1 \mathrm{~dB}, 3.5 \mathrm{~dB}$, $3.9 \mathrm{~dB}, 3.6 \mathrm{~dB}$, all $P<0.01$ ). For the older group, no significant differences in $40 \mathrm{~Hz}$ amplitudes were found between $\mathrm{NH}$ and $\mathrm{HI}$ listeners (all $P>0.18$ ).

When presenting the acoustic modulations at equal SPL (70 dB SPL), i.e., audibility was not corrected for, the SNR and amplitude analyses yielded the same results, without exception. We will therefore only report the outcomes of the SNR analysis. Note that, to reduce testing time, ASSR recordings at equal 
SPL were limited to $L$ stimulus presentation. In contrast to the equal loudness level conditions (Fig. 3), no differences in degree of neural synchronization to 4 and $40 \mathrm{~Hz}$ modulations were detected between $\mathrm{HI}$ and $\mathrm{NH}$ adults for any of the three age groups (all $P>0.05$ ) (Fig. 4). For the $80 \mathrm{~Hz}$ synchronization, we found a significant interaction between hearing status and age group $(F(2,83)=3.08, P=0.05)$. Enhanced synchronization to $80 \mathrm{~Hz}$ modulations, reflected by higher $80 \mathrm{~Hz}$ SNRs, was detected in $\mathrm{HI}$ young and middle-aged adults, but not in $\mathrm{HI}$ older participants (young: $M D=3.6 \mathrm{~dB}, P=0.016$; middleaged: $M D=4.6 \mathrm{~dB}, P<0.001$; older: $M D=0 \mathrm{~dB}, P=1.00$ ).

$<<$ insert Fig. 4 about here $>>$

It is to be noted that the effects of hearing status, except for the $40 \mathrm{~Hz}$ amplitude, showed no interaction with side of stimulation. Hence, hearing-related differences in degree of neural synchronization were considered to be robust.

\subsection{Hemispheric asymmetry}

When a significant majority of participants of a certain group were classified into the laterality category $\mathrm{LH}, \mathrm{RH}$ or symmetrical (SYM) (see 2.1.5), this group was considered to show a significant hemispheric asymmetry for processing of the acoustic modulation under investigation (Table 2). The hemispheric asymmetry for an acoustic modulation was not overall significant for the three sides of stimulation, which is in agreement with previous research (Poelmans et al., 2012). A number of differences in hemispheric asymmetry were identified between $\mathrm{HI}$ adults and $\mathrm{NH}$ controls, but the $\mathrm{RH}$ asymmetry for $40 \mathrm{~Hz}$ modulations in $\mathrm{HI}$ older adults compared to $\mathrm{NH}$ older persons who showed no significant asymmetry, was most striking because of two reasons. Firstly, the difference in hemispheric asymmetry for $40 \mathrm{~Hz}$ modulations in the older cohort was the only one apparent for the three sides of stimulation. Secondly, the analysis on the degree of neural synchronization only revealed a significant hearing-hemisphere interaction for the $40 \mathrm{~Hz}$ ASSR in the older cohort, i.e., HI older persons exhibited larger $40 \mathrm{~Hz}$ ASSRs in the $\mathrm{RH}$ compared to the $\mathrm{LH}$, while $\mathrm{NH}$ older adults showed equivalent ASSRs (Fig. 3).

When presenting the stimuli at equal SPL (70 dB SPL), the difference in $40 \mathrm{~Hz}$ hemispheric asymmetry between $\mathrm{HI}$ and $\mathrm{NH}$ older adults remained (Table 2). 


\begin{tabular}{|c|c|c|c|c|c|c|c|}
\hline & \multicolumn{2}{|c|}{ young } & \multicolumn{2}{|c|}{ middle-aged } & \multicolumn{2}{|c|}{ older } \\
\hline & & $\mathrm{NH}$ & $\mathrm{HI}$ & $\mathrm{NH}$ & $\mathrm{HI}$ & $\mathrm{NH}$ & $\mathrm{HI}$ \\
\hline $4 \mathrm{~Hz}$ & $\begin{array}{l}\mathrm{L} \\
\mathrm{R} \\
\mathrm{BI}\end{array}$ & $\mathrm{LH}$ & & $\mathrm{RH}$ & $\mathrm{RH}$ & & $\mathrm{RH}$ \\
\hline $20 \mathrm{~Hz}$ & $\begin{array}{l}\mathrm{L} \\
\mathrm{R} \\
\mathrm{BI}\end{array}$ & & & & & & \\
\hline $40 \mathrm{~Hz}$ & $\begin{array}{l}\mathrm{L} \\
\mathrm{R} \\
\mathrm{BI}\end{array}$ & $\mathrm{RH}$ & $\mathrm{RH}$ & & & & $\begin{array}{l}\mathrm{RH}^{*} \\
\mathrm{RH} \\
\mathrm{RH}\end{array}$ \\
\hline $80 \mathrm{~Hz}$ & $\begin{array}{l}\mathrm{L} \\
\mathrm{R} \\
\mathrm{BI}\end{array}$ & $\begin{array}{l}\mathrm{RH} \\
\mathrm{RH}\end{array}$ & & $\mathrm{RH}$ & $\mathrm{RH}$ & $\mathrm{RH}$ & \\
\hline
\end{tabular}

Table 2. Overview of the laterality categories (LH, RH, SYM) into which a significant majority of participants of a particular group $(\mathrm{NH} / \mathrm{HI}$ young, middle-aged, older) were classified for each acoustic envelope modulation $(4,20,40,80 \mathrm{~Hz})$ per side of stimulus presentation ( $\mathrm{L}, \mathrm{R}, \mathrm{BI})$ when presenting the stimuli at equal loudness levels. If no category is reported, the distribution among the three categories was similar. An asterisk indicates that the hemispheric asymmetry was observed in the equal loudness level as well as in the equal SPL ( $L$ only) condition.

\section{Discussion}

The aim of the present study was to characterize changes in neural envelope encoding related to hearing impairment in persons of different ages. By measuring ASSRs to different modulation frequencies, i.e., $4,20,40$, and $80 \mathrm{~Hz}$, we indirectly measured neural envelope encoding from the brainstem up to the cortex. We investigated the degree as well as the hemispheric asymmetry of neural envelope encoding. Adults belonging to three age groups were tested to examine whether associations between hearing impairment and neural envelope differed between different age groups. Also, the acoustic modulations were presented at equal loudness levels and at equal SPL to look into the effect of stimulus audibility.

Our data show that, when stimulus audibility is corrected for, neural synchronization in both subcortical and cortical auditory regions is significantly enhanced in young and middle-aged $\mathrm{HI}$ adults. When audibility is not corrected for, the enhancement is only observed in the brainstem. Interestingly, older $\mathrm{HI}$ adults do not show changes in the degree of neural synchronization relative to $\mathrm{NH}$ controls, however, they present with a significant synchronization asymmetry towards the $\mathrm{RH}$.

The enhanced neural synchronization in the brainstem of $\mathrm{HI}$ young and middle-aged adults is in agreement with observations in animal models (Henry et al., 2014; Kale and Heinz, 2010, 2012; Zhong et al., 2014) and with the study in middle-aged human listeners by Anderson et al. (2013). In addition to confirming that the degree of neural envelope encoding in the brainstem is independent of stimulus 
audibility, we extend Anderson and colleagues' findings by showing that increased subcortical neural synchronization is also present in $\mathrm{HI}$ young adults, though not in $\mathrm{HI}$ older individuals.

Our observation of enhanced neural synchronization at the cortical level is in accordance with the study by Millman et al. (2017). They tested NH and HI persons who were $\sim 60$ years of age and amplified the stimuli for their $\mathrm{HI}$ participants. Note that this set-up did not allow them to investigate variations related to age or audibility, while our set-up did allow for this detailed examination. In fact, we demonstrate that, similar to middle-aged $\mathrm{HI}$ adults, young $\mathrm{HI}$ adults exhibit enhanced neural synchronization in the auditory cortex, and that no such enhancement is present in older HI persons. Also, our data show an interplay between stimulus audibility and cortical neural synchronization. When correcting for audibility, $\mathrm{HI}$ young and middle-aged listeners show more neural synchronization at the cortical level, relative to $\mathrm{NH}$ controls. Yet, when the stimulus is presented at equal SPL, $\mathrm{HI}$ young and middle-aged individuals show a similar degree of neural synchronization as NH controls. This interplay between stimulus audibility and cortical neural synchronization is in line with behavioral research documenting that, compared to $\mathrm{NH}$ persons, $\mathrm{HI}$ listeners show higher sensitivity to envelope modulations (lower AM detection thresholds), when the modulations are presented at equal sensation levels, but not when they are presented at equal SPL (Bacon and Gleitman, 1992; Schlittenlacher and Moore, 2016).

A notable discrepancy in the present study concerns the unaffected processing of $20 \mathrm{~Hz}$ modulations as opposed to the enhanced neural synchronization to 4,40 , and $80 \mathrm{~Hz}$ modulations. Since both 20 and $40 \mathrm{~Hz}$ modulations are primarily processed near the thalamus (Giraud et al., 2000; Luke et al., 2017), the difference cannot be attributed to a distinct anatomical location for processing of $20 \mathrm{~Hz}$ modulations that would remain unaffected in $\mathrm{HI}$ individuals. The reason for the discrepancy thus remains unclear.

The enhanced neural synchronization in $\mathrm{HI}$ listeners can be explained by homeostatic mechanisms. It has been demonstrated that the reduced cochlear output in $\mathrm{HI}$ individuals following hair cell loss and/or synaptopathy, triggers various mechanisms that induce central gain to sustain an operative level of neural excitability (Kujawa and Liberman, 2015; Salvi et al., 2017). For instance, HI auditory nerve fibers show steeper-than-normal response growths with acoustic input level (Heinz and Young, 2003; Kale and Heinz, 2010), enhanced onset responses (Crumling and Saunders, 2007), and slower recovery from neural adaptation (Scheidt et al., 2010). Moreover, from the HI brainstem up to the 
cortex, the influx of inhibitory neurotransmitters into excitatory neurons is reduced, while it is preserved for inhibitory neurons (Sanes and Kotak, 2011; Sarro et al., 2008; Vale and Sanes, 2002).

Despite a compensatory increase in neural synchronization, higher sensitivity to envelope modulations in $\mathrm{HI}$ persons is thought to be negatively corrrelated with speech perception. This theory is supported by behavioral research showing poorer speech perception performance, especially in the presence of a fluctuating background noise, when perceptually enhancing the envelope modulations of the target speech (Moore and Glasberg, 1993; Moore et al., 1995). Likewise, electrophysiological research has indicated that a higher degree of neural envelope encoding at the cortical level correlates with poorer speech understanding in a modulated masking noise (Millman et al., 2017). The correspondence between enhanced sensitivity to envelope modulations and degraded speech perception might be attributed to an envelope-to-TFS processing imbalance. Adequate processing of the speech envelope is required yet not sufficient for the perception of masked speech: the TFS needs to be adequately processed as well (Lorenzi et al., 2006; Peelle and Davis, 2012). Neuroimaging studies have found neural TFS encoding to be reduced in $\mathrm{HI}$ adults relative to $\mathrm{NH}$ controls (Ananthakrishnan et al., 2016; Vercammen et al., 2018) and behavioral research has demonstrated that $\mathrm{HI}$ listeners show poor sensitivity to TFS information (Buss et al., 2004). Taken together with the observations of enhanced neural encoding and behavioral detection of envelope modulations in $\mathrm{HI}$ listeners, hearing impairment appears to be accompanied by a marked imbalance between processing of envelope and TFS cues, which is assumed to be detrimental for speech perception. In this framework, further research is warranted to clarify whether the poorer speech perception with enhanced envelope encoding concerns either a one-to-one relationship or an indirect relationship mediated by TFS deficits, i.e., envelope enhancement acting as a compensatory strategy.

Altogether, young and middle-aged $\mathrm{HI}$ adults show enhanced neural synchronization to envelope modulations, especially when stimulus audibility is corrected for. Importantly, audibility correction by hearing aids currently constitutes the basis of rehabilitation strategies to improve speech perception. As it is presumed that enhanced neural envelope encoding is related to worse speech perception, one could argue that the amplification provided by hearing aids may not always be beneficial for $\mathrm{HI}$ listeners, especially not in listening situations with fluctuating background noises. Further research is required to explore this idea. 
In contrast to young and middle-aged $\mathrm{HI}$ persons, older $\mathrm{HI}$ adults do not show enhanced neural synchronization to envelope modulations. The reason for this age-related variation remains to be determined. According to the study by Kale and Heinz (2010), the increase in neural synchronization correlates with the degree of hearing impairment. In effect, these authors demonstrated that higher threshold shifts correspond to more enhanced envelope encoding in auditory nerve fibers. In the present study, however, the degree of hearing impairment does not yield an adequate explanation for the observed age-related disparity. The threshold shift, calculated as PTA $125-8000 \mathrm{~Hz} \mathrm{HI}-\mathrm{PTA} 125-8000 \mathrm{~Hz}$ $\mathrm{NH}$, was, on average, significantly smaller in the older group (33 dB HL) than in the young group (47 $\mathrm{dB} \mathrm{HL}$ ), yet it was similar to the threshold shift in the middle-aged group (39 dB HL). Another possible explanation for the age-related variation could lie in neural plasticity, which is a key feature of the human brain. Auditory plasticity refers to morphological and physiological alterations in the central nervous system due to changes in the auditory environment. There is a large body of evidence showing altered neural behavior (e.g., neural synchronization) in the auditory brain following hearing impairment or hearing aid use, causing auditory deprivation and amplification, respectively (Neuman, 2005; Munro, 2008). Nonetheless, we do not find our older HI participants to differ significantly from both the young and middle-aged $\mathrm{HI}$ participants with respect to three important environmental factors related to auditory plasticity, i.e., having hearing impairment, using hearing aids, and being auditory deprived (Table 3).

\begin{tabular}{|c|c|c|c|c|c|c|}
\hline & heal & impairment & heari & use & aud & ation \\
\hline & mea & SD & mear & & mea & \\
\hline young & $24 \pm$ & & $21 \pm$ & & $3 \pm$ & \\
\hline middle-aged & $18 \pm$ & & $10 \pm$ & & $8 \pm$ & \\
\hline older & $12 \pm$ & & $7 \pm$ & & $5 \pm$ & \\
\hline & heal & impairment & heari & use & aud & ation \\
\hline & MD & $P^{1}$ & MD & $P^{1}$ & MD & $P^{2}$ \\
\hline older vs. young & 12 & 0.002 & 14 & $<0.001$ & 2 & 0.467 \\
\hline older vs. middle-aged & 6 & 0.132 & 3 & 0.815 & 3 & 0.514 \\
\hline
\end{tabular}

Table 3. The mean duration \pm SD (in years) of three factors related to auditory plasticity, i.e., having hearing impairment, using hearing aids, and being auditory deprived, for the HI young, middle-aged, and older participants are summarized in the upper part of the table. The duration of auditory deprivation was calculated by subtracting the duration of hearing aid use from the duration of having hearing impairment. For each factor, a one-way ANOVA and post hoc testing was performed for comparing older to young and middle-aged listeners: the MDs (in years) and $P$ values are outlined in the lower part of the table.

${ }^{1}$ Bonferroni corrected; ${ }^{2}$ Games-Howell corrected

Although the older $\mathrm{HI}$ adults had been $\mathrm{HI}$ and had been using hearing aids for a shorter period of time than the young $\mathrm{HI}$ adults, no such differences were apparent between older and middle-aged $\mathrm{HI}$ 
participants. Moreover, $\mathrm{HI}$ older participants had been auditory deprived for the same amount of time as $\mathrm{HI}$ young and middle-aged participants.

Rather than the degree of hearing impairment or factors related to neural plasticity, the etiology of hearing impairment and/or altered neural envelope encoding at an older age could account for the divergent outcomes in the older compared to the young and middle-aged adults. Recall that, although all participants had hearing loss that was sensorineural in nature, the etiology of hearing impairment was different for the three age groups. Hearing loss at birth or following severe/chronic otitis was the case for the young adults, familial hearing loss was indicated in the HI middle-aged participants, and all older persons were diagnosed with presbycusis. Given that the hearing impairment of older adults is not associated with enhanced neural envelope encoding, as opposed to the hearing impairment of young and middle-aged adults, the present study suggests that presbycusis, in contrast to hearing losses of other origins, is not associated with enhanced neural envelope encoding. An important note to make is that different mechanisms are associated with presbycusis, including metabolic changes, loss of outer hair cells, and loss of afferent nerve fibers (for a review see Gordon-Salant et al., 2010). It would be interesting to implement diagnostic measurements to differentiate between different types of presbycusis among HI older participants (e.g., Verhulst et al., 2016), in order to investigate whether there is an interplay between neural envelope encoding and type of presbycusis. Altered neural envelope encoding at an older age may also explain the absence of increased neural synchronization in older adults at the cortical level, and particularly to $4 \mathrm{~Hz}$ envelope modulations. In a previous study (Goossens et al., 2016), we observed a higher degree of neural synchronization to $4 \mathrm{~Hz}$ envelope modulations in older $\mathrm{NH}$ persons compared to young and middle-aged $\mathrm{NH}$ individuals. This observation is in line with other studies indicating enhanced cortical envelope encoding at an older age, independent of hearing impairment (Bidelman et al., 2014; Presacco et al., 2016; Sörös et al., 2009). This age-related enhancement can be attributed to the previously described homeostatic mechanisms, since, similarly to hearing impairment, aging goes with a decrease in cochlear output (Sergeyenko et al., 2013). Hence, at an older age, homeostatic mechanisms are activated, independent of hearing sensitivity. Taken together with the current observation that hearing impairment at an older age does not relate to increased cortical synchronization, we presume that the homeostatic potential is maximally exploited at an older age. As a consequence, no (further) increase in cortical envelope encoding is detected for hearing impairment at an older age, in contrast to hearing 
impairment at a young and middle age. In sum, presbycusis and older age being characterized by altered envelope encoding, are the most plausible explanations for the discrepant observations in older versus young and middle-aged adults. Disentangling their differential contribution is, however, not possible, since presbycusis and older age are intrinsically related to each other.

Interestingly, the older group shows another striking difference with the young and middle-aged group. While no substantial differences in hemispheric asymmetry are detected between $\mathrm{HI}$ and $\mathrm{NH}$ young and middle-aged individuals, $\mathrm{HI}$ and $\mathrm{NH}$ older adults show a marked difference in hemispheric asymmetry. For $40 \mathrm{~Hz}$ modulations, $\mathrm{HI}$ older persons show a significant synchronization asymmetry towards the $\mathrm{RH}$, while no such hemispheric asymmetry is present in $\mathrm{NH}$ older persons. To our knowledge, this study is the first to look into the association between hearing impairment and functional hemispheric asymmetry. Previously, Bellis et al. (2000) documented that young NH adults show greater neural synchronization to speech syllables in the $\mathrm{LH}$ compared to the $\mathrm{RH}$, while older $\mathrm{NH}$ adults show a similar degree of neural synchronization in both hemispheres. This altered hemispheric asymmetry with age is in agreement with the evidence-based HAROLD model (Cabeza, 2002), which states that neural processing is less asymmetrical in older compared to young adults. Although aging is thus thought to be characterized by less asymmetrical neural processing, our HI older participants exhibit a clear asymmetrical processing pattern. This novel observation indicates that presbycusis interferes with age-related variations in functional hemispheric asymmetry, which may be explained by the reduced integrity of white matter tracts following presbycusis (Mudar and Husain, 2016). The corpus callosum, which plays a predominant role in interhemispheric interactions (Hoptman and Davidson, 1994), is a large bundle of white matter tracts. As such, white matter deficits in the presbyacusic brain can impact the hemispheric asymmetry in neural synchronization. Intriguingly, research on functional hemispheric asymmetry and speech intelligibility suggests that persons who show an asymmetrical processing pattern have better speech perception than persons who show a symmetrical pattern (Bellis et al., 2000; Hämäläinen et al., 2012). Because of the significant speech perception difficulties of $\mathrm{HI}$ older persons, it seems, however, unlikely that their $\mathrm{RH}$ asymmetry would have a beneficial effect on speech perception. Further research is needed to clarify the contribution of this $\mathrm{RH}$ asymmetry to speech perception. 


\section{Conclusions}

In conclusion, this study demonstrates that hearing impairment is characterized by changes in neural envelope encoding, the nature of which is different in young and middle-aged compared to older persons. Hearing impairment in young and middle-aged adults is characterized by enhanced neural envelope encoding from the brainstem up to the cortex, which is most pronounced when stimulus audibility is corrected for. Importantly, such enhanced envelope encoding is thought to underlie impaired speech perception. In older adults, hearing impairment (presbycusis) is not associated with enhanced neural envelope encoding, yet it coincides with a clear hemispheric asymmetry in neural synchronization, which may act on speech perception as well.

These are crucial insights into a key neural mechanism underlying speech perception in $\mathrm{HI}$ persons that can contribute to the development of advanced intervention strategies to remediate impaired speech perception.

\section{Acknowledgments}

Our special thanks go to all participants. We are grateful to our master's students for their help with data collection, to Jonas Vanthornhout, Michael Hofmann, and Robert Luke for their technical support, to Astrid De Vos for fruitful discussions on the data analysis, and to An Billiau, Celsus Medical Writing LLC, for editing assistance to this manuscript.

This research was funded by the Research Foundation - Flanders (FWO) through an FWO-aspirant grant to Tine Goossens (grant number 11Z8817N) and by the Research Council of KU Leuven (project OT/12/98).

Declarations of interest: none 


\section{References}

Ahissar, E., Nagarajan, S., Ahissar, M., Protopapas, A., Mahncke, H., Merzenich, M.M., 2001. Speech comprehension is correlated with temporal response patterns recorded from auditory cortex. Proc. Natl. Acad. Sci. U. S. A. 98, 13367-13372. doi:10.1073/pnas.201400998

Ananthakrishnan, S., Krishnan, A., Bartlett, E., 2016. Human frequency following response: neural representation of envelope and temporal fine structure in listeners with normal hearing and sensorineural hearing loss. Ear Hear. 37, e91-e103. doi:10.1097/AUD.0000000000000247

Anderson, S., Parbery-Clark, A., White-Schwoch, T., Drehobl, S., Kraus, N., 2013. Effects of hearing loss on the subcortical representation of speech cues. J. Acoust. Soc. Am. 133, 3030-3038. doi:10.1121/1.4799804

Anderson, S., Parbery-Clark, A., White-Schwoch, T., Kraus, N., 2012. Aging affects neural precision of speech encoding. J. Neurosci. 32, 14156-14164. doi:10.1523/JNEUROSCI.2176-12.2012

Anderson, S., Parbery-Clark, A., Yi, H.-G., Kraus, N., 2011. A neural basis of speech-in-noise perception in older adults. Ear Hear. 32, 750-757. doi:10.1097/AUD.0b013e31822229d3

Ansari, M.S., Rangasayee, R., Ansari, M.A.H., 2017. Neurophysiological aspects of brainstem processing of speech stimuli in audiometric-normal geriatric population. J. Laryngol. Otol. 131, 239-244. doi:10.1017/S0022215116009841

Bland, J.M., Altman, D.G., 1994. Correlation, regression, and repeated data. BMJ 308, 896. doi:10.1136/bmj.308.6942.1510a

Brame, R., Paternoster, R., Mazerolle, P., Piquero, A., 1998. Testing for the equality of maximumlikelihood regression coefficients between two independent equations. J. Quant. Criminol. 14, 245261. doi:10.1023/A:1023030312801

Bregman, A.S., 1990. Auditory scene analysis: the perceptual organization of sounds, 1st ed. MIT Press, Cambridge, MA.

Brungart, D.S., 2001. Informational and energetic masking effects in the perception of two simultaneous talkers. J. Acoust. Soc. Am. 109, 1101-1109. doi:10.1121/1.1345696

Buss, E., Hall, J.W., Grose, J.H., 2004. Temporal fine-structure cues to speech and pure tone modulation in observers with sensorineural hearing loss. Ear Hear. 25, 242-250. doi:10.1097/01.AUD.0000130796.73809.09

Chait, M., Greenberg, S., Arai, T., Simon, J.Z., Poeppel, D., 2015. Multi-time resolution analysis of 
speech:

evidence from psychophysics. Front. Neurosci. 9, 214. doi:10.3389/fnins.2015.00214

Christiansen, C., Dau, T., 2012. Relationship between masking release in fluctuating maskers and speech reception thresholds in stationary noise. J. Acoust. Soc. Am. 132, 1655-1666. doi:10.1121/1.4742732

Coffey, E.B.J., Herholz, S.C., Chepesiuk, A.M.P., Baillet, S., Zatorre, R.J., 2016. Cortical contributions to the auditory frequency-following response revealed by MEG. Nat. Commun. 7, 11070. doi:10.1038/ncomms 11070

Coffey, E.B.J., Musacchia, G., Zatorre, R.J., 2017. Cortical correlates of the auditory frequency-following and onset responses: EEG and fMRI evidence. J. Neurosci. 37, 830-838. doi:10.1523/JNEUROSCI.1265-16.2017

Cohen, A., 1983. Comparing regression coefficients across subsamples: a study of the statistical test. Sociol. Methods Res. 12, 77-94. doi:10.1177/0049124183012001003

Dimitrijevic, A., John, M.S., Picton, T.W., 2004. Auditory steady-state responses and word recognition scores in normal-hearing and hearing-impaired adults. Ear Hear. 25, 68-84. doi:10.1097/01.AUD.0000111545.71693.48

Ding, N., Simon, J.Z., 2012. Emergence of neural encoding of auditory objects while listening to competing speakers. Proc. Natl. Acad. Sci. U. S. A. 109, 5-10. doi:10.1073/pnas.1205381109

Doelling, K.B., Arnal, L.H., Ghitza, O., Poeppel, D., 2014. Acoustic landmarks drive delta-theta oscillations to enable speech comprehension by facilitating perceptual parsing. Neuroimage 85 , 761-768. doi:10.1016/j.neuroimage.2013.06.035

Drullman, R., Festen, J.M., Plomp, R., 1994. Effect of temporal envelope smearing on speech reception. J. Acoust. Soc. Am. 95, 1053-1064. doi:10.1121/1.408467

Dubno, J.R., Horwitz, A.R., Ahlstrom, J.B., 2002. Benefit of modulated maskers for speech recognition by younger and older adults with normal hearing. J. Acoust. Soc. Am. 111, 2897-2907. doi:10.1121/1.1480421

Durlach, N.I., Mason, C.R., Kidd, G., Arbogast, T.L., Colburn, H.S., Shinn-Cunningham, B.G., 2003. Note on informational masking. J. Acoust. Soc. Am. 113, 2984-2987. doi:10.1121/1.1570435

Edwards, E., Chang, E.F., 2013. Syllabic $(\sim 2-5 \mathrm{~Hz})$ and fluctuation $(\sim 1-10 \mathrm{~Hz})$ ranges in speech and auditory processing. Hear. Res. 305, 113-134. doi:10.1016/j.heares.2013.08.017 
Emara, A.A.Y., Kolkaila, E.A., 2010. Prediction of loudness growth in subjects with sensorineural hearing loss using auditory steady state response. J. Int. Adv. Otol. 6, 371-379.

Festen, J.M., Plomp, R., 1990. Effects of fluctuating noise and interfering speech on the speechreception threshold for impaired and normal hearing. J. Acoust. Soc. Am. 88, 1725-1736. doi:10.1121/1.400247

Francart, T., van Wieringen, A., Wouters, J., 2011. Comparison of fluctuating maskers for speech recognition tests. Int. J. Audiol. 50, 2-13. doi:10.3109/14992027.2010.505582

Füllgrabe, C., Moore, B.C.J., Stone, M.A., 2015. Age-group differences in speech identification despite matched audiometrically normal hearing: contributions from auditory temporal processing and cognition. Front. Aging Neurosci. 6, 347. doi:10.3389/fnagi.2014.00347

Giraud, A.-L., Lorenzi, C., Ashburner, J., Wable, J., Johnsrude, I., Frackowiak, R., Kleinschmidt, A., 2000. Representation of the temporal envelope of sounds in the human brain. J. Neurophysiol. 84 , 1588-1598. doi:10.1152/jn.2000.84.3.1588

Goossens, T., Vercammen, C., Wouters, J., van Wieringen, A., 2017. Masked speech perception across the adult lifespan: impact of age and hearing impairment. Hear. Res. 344, 109-124. doi:10.1016/j.heares.2016.11.004

Goossens, T., Vercammen, C., Wouters, J., van Wieringen, A., 2016. Aging affects neural synchronization to speech-related acoustic modulations. Front. Aging Neurosci. 8, 133. doi:10.3389/fnagi.2016.00133

Goossens, T., Vercammen, C., Wouters, J., van Wieringen, A. The impact of hearing impairment on neural envelope encoding at different ages. under review

Gourévitch, B., Edeline, J.M., Occelli, F., Eggermont, J.J., 2014. Is the din really harmless? Long-term effects of non-traumatic noise on the adult auditory system. Nat. Rev. Neurosci. 15, 483-491. doi:10.1038/nrn3744

Greenberg, S., Carvey, H., Hitchcock, L., Chang, S., 2003. Temporal properties of spontaneous speech—a syllable-centric perspective. J. Phon. 31, 465-485. doi:10.1016/j.wocn.2003.09.005

Grose, J.H., Mamo, S.K., Hall, J.W., 2009. Age effects in temporal envelope processing: speech unmasking and auditory steady state responses. Ear Hear. 30, 568-575. doi:10.1097/AUD.0b013e3181ac128f

Hastie, T., Tibshirani, R., Friedman, J., 2009. Linear methods for regression, in: Hastie, T., Tibshirani, 
R., Friedman, J. (Eds.), The elements of statistical learning: data mining, inference, and prediction. Springer, New York, NY, pp. 43-100.

Helfer, K.S., Wilber, L.A., 1990. Hearing loss, aging, and speech perception in reverberation and noise. J. Speech Hear. Res. 33, 149-155. doi:10.1044/jshr.3301.149

Herdman, A.T., Lins, O., Van Roon, P., Stapells, D.R., Scherg, M., Picton, T.W., 2002. Intracerebral sources of human auditory steady-state responses. Brain Topogr. 15, 69-86. doi:10.1023/A:1021470822922

Holube, I., Fredelake, S., Vlaming, M., Kollmeier, B., 2010. Development and analysis of an International Speech Test Signal (ISTS). Int. J. Audiol. 49, 891-903. doi:10.3109/14992027.2010.506889

Hopkins, K., Moore, B.C.J., 2011. The effects of age and cochlear hearing loss on temporal fine structure sensitivity, frequency selectivity, and speech reception in noise. J. Acoust. Soc. Am. 130, 334349. doi:10.1121/1.3585848

Hopkins, K., Moore, B.C.J., 2009. The contribution of temporal fine structure to the intelligibility of speech in steady and modulated noise. J. Acoust. Soc. Am. 125, 442-446. doi:10.1121/1.3037233

Humes, L.E., Roberts, L., 1990. Speech-recognition difficulties of the hearing-impaired elderly: the contributions of audibility. J. Speech Hear. Res. 33, 726-735. doi:10.1044/jshr.3304.726

International Organization for Standardization, 1998. ISO 389-1: Acoustics - Reference zero for the calibration of audiometric equipment. Part 1: Reference equivalent threshold sound pressure levels for pure tones and supra-aural earphones. Geneva, Switzerland.

Jansen, S., Luts, H., Wagener, K.C., Kollmeier, B., Del Rio, M., Dauman, R., James, C., Fraysse, B., Vormès, E., Frachet, B., Wouters, J., van Wieringen, A., 2012. Comparison of three types of French speech-in-noise tests: a multi-center study. Int. J. Audiol. 51, 164-173. doi:10.3109/14992027.2011.633568

Joris, P.X., Schreiner, C.E., Rees, A., 2004. Neural processing of amplitude-modulated sounds. Physiol. Rev. 84, 541-577. doi:10.1152/physrev.00029.2003

Kale, S., Heinz, M.G., 2010. Envelope coding in auditory nerve fibers following noise-induced hearing loss. J. Assoc. Res. Otolaryngol. 11, 657-673. doi:10.1007/s10162-010-0223-6

Kidd, G., Mason, C.R., Deliwala, P.S., Woods, W.S., Colburn, H.S., 1994. Reducing informational masking by sound segregation. J. Acoust. Soc. Am. 95, 3475-3480. doi:10.1121/1.410023

Kujawa, S.G., Liberman, M.C., 2015. Synaptopathy in the noise-exposed and aging cochlea: primary 
neural degeneration in acquired sensorineural hearing loss. Hear. Res. 330, 191-199. doi:10.1016/j.heares.2015.02.009

Kujawa, S.G., Liberman, M.C., 2009. Adding insult to injury: cochlear nerve degeneration after "temporary" noise-induced hearing loss. J. Neurosci. 29, 14077-14085. doi:10.1523/JNEUROSCI.2845-09.2009

Kwon, B.J., Turner, C.W., 2001. Consonant identification under maskers with sinusoidal modulation: masking release or modulation interference? J. Acoust. Soc. Am. 110, 1130-1140. doi:10.1121/1.1384909

Leigh-Paffenroth, E.D., Fowler, C.G., 2006. Amplitude-modulated auditory steady-state responses in younger and older listeners. J. Am. Acad. Audiol. 17, 582-597. doi:10.3766/jaaa.17.8.5

Lopes da Silva, F., 2013. EEG and MEG: relevance to neuroscience. Neuron 80, 1112-1128. doi:10.1016/j.neuron.2013.10.017

Lorenzi, C., Gilbert, G., Carn, H., Garnier, S., Moore, B.C.J., 2006. Speech perception problems of the hearing impaired reflect inability to use temporal fine structure. Proc. Natl. Acad. Sci. U. S. A. 103, 18866-18869. doi:10.1073/pnas.0607364103

Luke, R., De Vos, A., Wouters, J., 2017. Source analysis of auditory steady-state responses in acoustic and electric hearing. Neuroimage 147, 568-576. doi:10.1016/j.neuroimage.2016.11.023

Ménard, M., Gallégo, S., Berger-Vachon, C., Collet, L., Thai-Van, H., 2008. Relationship between loudness growth function and auditory steady-state response in normal-hearing subjects. Hear. Res. 235, 105-113. doi:10.1016/j.heares.2007.10.007

Millman, R.E., Mattys, S.L., Gouws, A.D., Prendergast, G., 2017. Magnified neural envelope coding predicts deficits in speech perception in noise. J. Neurosci. 37, 7727-7736. doi:10.1523/JNEUROSCI.2722-16.2017

Moore, B.C.J., Glasberg, B.R., Vickers, D.A., 1995. Simulation of the effects of loudness recruitment on the intelligibility of speech in noise. Br. J. Audiol. 29, 131-143. doi:10.3109/03005369509086590

Moore, B.C.J., 2014. Auditory processing of temporal fine structure: effects of age and hearing loss, $1 \mathrm{st}$ ed. World Scientific Publishing CO. Pte. Ltd., Toh Tuck, Singapore.

Moore, B.C.J., 2007. Cochlear hearing loss: physiological, psychological and technical issues, 2 nd ed. Wiley, Chichester, UK.

Moore, B.C.J., Glasberg, B.R., 1993. Simulation of the effects of loudness recruitment and threshold 
elevation on the intelligibility of speech in quiet and in a background of speech. J. Acoust. Soc. Am. 94, 2050-2062. doi:10.1121/1.407478

Moore, B.C.J., Wojtczak, M., Vickers, D.A., 1996. Effect of loudness recruitment on the perception of amplitude modulation. J. Acoust. Soc. Am. 100, 481-489. doi:10.1121/1.415861

Nasreddine, Z.S., Phillips, N.A., Bédirian, V., Charbonneau, S., Whitehead, V., Collin, I., Cummings, J.L., Chertkow, H., 2005. The Montreal Cognitive Assessment, MoCA: a brief screening tool for mild cognitive impairment. J. Am. Geriatr. Soc. 53, 695-9. doi:10.1111/j.1532-5415.2005.53221.x

Okamoto, H., Stracke, H., Draganova, R., Pantev, C., 2009. Hemispheric asymmetry of auditory evoked fields elicited by spectral versus temporal stimulus change. Cereb. Cortex 19, 2290-2297. doi:10.1093/cercor/bhn245

Oldfield, R.C., 1971. The assessment and analysis of handedness: the Edinburgh inventory. Neuropsychologia 9, 97-113. doi:10.1016/0028-3932(71)90067-4

Peelle, J.E., Davis, M.H., 2012. Neural oscillations carry speech rhythm through to comprehension. Front. Psychol. 3, 320. doi:10.3389/fpsyg.2012.00320

Plack, C.J., Barker, D., Prendergast, G., 2014. Perceptual consequences of "hidden" hearing loss. Trends Hear. 18, 1-11. doi:10.1177/2331216514550621

Plomp, R., Mimpen, A.M., 1979. Improving the reliability of testing the speech reception threshold for sentences. Audiology 18, 43-52. doi:10.3109/00206097909072618

Poeppel, D., 2003. The analysis of speech in different temporal integration windows: cerebral lateralization as "asymmetric sampling in time." Speech Commun. 41, 245-255. doi:10.1016/S0167-6393(02)00107-3

Presacco, A., Jenkins, K., Lieberman, R., Anderson, S., 2015. Effects of aging on the encoding of dynamic and static components of speech. Ear Hear. 36, e352-e363. doi:10.1097/AUD.0000000000000193

Presacco, A., Simon, J.Z., Anderson, S., 2016. Evidence of degraded representation of speech in noise, in the aging midbrain and cortex. J. Neurophysiol. 116, 2346-2355. doi:10.1152/jn.00372.2016

Purcell, D.W., John, S.M., Schneider, B.A., Picton, T.W., 2004. Human temporal auditory acuity as assessed by envelope following responses. J. Acoust. Soc. Am. 116, 3581-3593. doi:10.1121/1.1798354

Rance, G., 2008. Auditory steady-state response: generation, recording, and clinical applications, 1st 
ed. Plural Publishing Inc, San Diego, CA.

Rosen, S., 1992. Temporal information in speech: acoustic, auditory and linguistic aspects. Philos. Trans. Biol. Sci. 336, 367-373. doi:10.1098/rstb.1992.0070

Schaette, R., McAlpine, D., 2011. Tinnitus with a normal audiogram: physiological evidence for hidden hearing loss and computational model. J. Neurosci. 31, 13452-13457. doi:10.1523/JNEUROSCI.2156-11.2011

Schlittenlacher, J., Moore, B.C.J., 2016. Discrimination of amplitude-modulation depth by subjects with normal and impaired hearing. J. Acoust. Soc. Am. 140, 3487-3495. doi:10.1121/1.4966117

Schoof, T., Rosen, S., 2016. The role of age-related declines in subcortical auditory processing in speech perception in noise. J. Assoc. Res. Otolaryngol. 17, 441-460. doi:10.1007/s10162-0160564-x

Schoonhoven, R., Boden, C.J.R., Verbunt, J.P.A., de Munck, J.C., 2003. A whole head MEG study of the amplitude-modulation-following response: phase coherence, group delay and dipole source analysis. Clin. Neurophysiol. 114, 2096-2106. doi:10.1016/S1388-2457(03)00200-1

Sek, A., Baer, T., Crinnion, W., Springgay, A., Moore, B.C.J., 2015. Modulation masking within and across carriers for subjects with normal and impaired hearing. J. Acoust. Soc. Am. 138, 11431153. doi:10.1121/1.4928135

Sergeyenko, Y., Lall, K., Liberman, M.C., Kujawa, S.G., 2013. Age-related cochlear synaptopathy: an early-onset contributor to auditory functional decline. J. Neurosci. 33, 13686-13694. doi:10.1523/JNEUROSCI.1783-13.2013

Shannon, R. V, Zeng, F.-G., Kamath, V., Wygonski, J., Ekelid, M., 1995. Speech recognition with primarily temporal cues. Science 270, 303-304. doi:10.1126/science.270.5234.303

Shinn-Cunningham, B.G., 2008. Object-based auditory and visual attention. Trends Cogn. Sci. 12, 182186. doi:10.1016/j.tics.2008.02.003

Souza, P.E., Turner, C.W., 1994. Masking of speech in young and elderly listeners with hearing loss. J. Speech Hear. Res. 37, 655-661. doi:10.1044/jshr.3703.655

Stone, M.A., Füllgrabe, C., Moore, B.C.J., 2012. Notionally steady background noise acts primarily as a modulation masker of speech. J. Acoust. Soc. Am. 132, 317-326. doi:10.1121/1.4725766

Stone, M.A., Moore, B.C.J., 2014. On the near non-existence of "pure" energetic masking release for speech. J. Acoust. Soc. Am. 135, 1967-1977. doi:10.1121/1.4868392 
Studenmund, A.H., Cassidy, H.J., 1987. Using econometrics: a practical guide, 1st ed. Little, Brown Book Group, London, UK.

Tlumak, A.I., Durrant, J.D., Delgado, R.E., 2015. The effect of advancing age on auditory middle- and long-latency evoked potentials using a steady-state-response approach. Am. J. Audiol. 24, 494507. doi:10.1044/2015_AJA-15-0036

Vale, C., Sanes, D.H., 2002. The effect of bilateral deafness on excitatory and inhibitory synaptic strength in the inferior colliculus. Eur. J. Neurosci. 16, 2394-2404. doi:10.1046/j.14609568.2002.02302.x

Van Eeckhoutte, M., Wouters, J., Francart, T., 2016. Auditory steady-state responses as neural correlates of loudness growth. Hear. Res. 342, 58-68. doi:10.1016/j.heares.2016.09.009

van Wieringen, A., Wouters, J., 2008. LIST and LINT: Sentences and numbers for quantifying speech understanding in severely impaired listeners for Flanders and the Netherlands. Int. J. Audiol. 47, 348-355. doi:10.1080/14992020801895144

Vercammen, C., Goossens, T., Undurraga, J., Wouters, J., van Wieringen, A. Electrophysiological and behavioral evidence of reduced binaural temporal processing in the aging and hearing impaired

human auditory system. Trends Hear. accepted for publication.

Wallaert, N., Moore, B.C.J., Ewert, S.D., Lorenzi, C., 2017. Sensorineural hearing loss enhances auditory sensitivity and temporal integration for amplitude modulation. J. Acoust. Soc. Am. 141, 971-980. doi:10.1121/1.4976080

Wang, X., Lu, T., Snider, R.K., Liang, L., 2005. Sustained firing in auditory cortex evoked by preferred stimuli. Nature 435, 341-346. doi:10.1038/nature03565

Wang, Y., Ding, N., Ahmar, N., Xiang, J., Poeppel, D., Simon, J.Z., 2012. Sensitivity to temporal modulation rate and spectral bandwidth in the human auditory system: MEG evidence. J. Neurophysiol. 107, 2033-2041. doi:10.1152/jn.00310.2011

Yost, W.A., Sheft, S., Opie, J., 1989. Modulation interference in detection and discrimination of amplitude modulation. J. Acoust. Soc. Am. 86, 2138-2147. doi:10.1121/1.398474

Zatorre, R.J., Belin, P., 2001. Spectral and temporal processing in human auditory cortex. Cereb. Cortex 11, 946-953. doi:10.1093/cercor/11.10.946

Zatorre, R.J., Belin, P., Penhune, V.B., 2002. Structure and function of auditory cortex: music and 
speech. Trends Cogn Sci 6, 37-46. doi:10.1016/s1364-6613(00)01816-7 


\section{Figure captions}

Fig. 1. Median audiometric thresholds ( $\mathrm{dB} \mathrm{HL}$ ) of $\mathrm{NH}$ (black) and $\mathrm{HI}$ (gray) participants, averaged across both ears. Thresholds are illustrated by diamonds, squares, and circles for young, middle-aged, and older persons, respectively. Errors bars indicate the interquartile range.

Fig. 2. Overview of the research design of the present study.

Fig. 3. Overview of the significant differences in degree of neural synchronization when presenting the acoustic modulations at equal loudness levels. The 4 and $80 \mathrm{~Hz}$ SNRs are shown in the top panels. The $40 \mathrm{~Hz}$ SNR and $40 \mathrm{~Hz}$ amplitude are displayed in the bottom panels. The $20 \mathrm{~Hz}$ ASSRs are not displayed, since no significant effects were found for this frequency. The dotted line in the SNR figures indicates the significance cut-off, i.e., $4.8 \mathrm{~dB}$. In each panel, age groups (young, middle-aged, older) are displayed per row, and hemispheres per column $(\mathrm{LH}, \mathrm{RH})$. The bars are clustered per hearing status $(\mathrm{NH}, \mathrm{HI})$ and represent the mean ASSR magnitude per side of stimulation, i.e., left ear $(\mathrm{L})$ (dark fill), right ear (R) (no fill), and bilaterally (BI) (gray fill). Error bars indicate the $95 \%$ confidence interval. $0.05<\# P<0.06 ;{ }^{*} P \leq 0.05 ;{ }^{* *} P \leq 0.01 ;{ }^{* * *} P \leq 0.001$.

The explanation for the discrepant effects in the SNR and amplitude of the $40 \mathrm{~Hz}$ ASSR resides in the induced EEG noise. $\mathrm{HI}$ young and middle-aged persons exhibited more EEG noise at $40 \mathrm{~Hz}$ than $\mathrm{NH}$ controls (both $P<0.05)$ and more $40 \mathrm{~Hz}$ noise correlated with lower $40 \mathrm{~Hz}$ SNRs $\left(r_{\mathrm{SNR} \text { noise }}=-0.40, P=\right.$ 0.047). When this correlation was controlled for, the $40 \mathrm{~Hz}$ SNR of young and middle-aged adults increased significantly with increasing PTA $125 \mathrm{~Hz}-8 \mathrm{kHz}\left(\mathrm{rPTA}_{\mathrm{P} \text { (SNR.noise) }}=0.47, P=0.013\right)$, similarly to the hearing-related increase in the $40 \mathrm{~Hz}$ amplitude ( $\left.r_{\mathrm{PTA} \text { ampl }}=0.50, P=0.016\right)$. 
Fig. 4. Overview of the degree of neural synchronization to the 4,40 , and $80 \mathrm{~Hz}$ acoustic envelope modulations presented at equal SPL (70 dB SPL), expressed in SNR (dB). Because equal SPL conditions were restricted to $L$ stimulus presentation, no distinction is made between side of stimulation. Similarly to the equal loudness conditions (Fig. 3), no significant effects were observed for the $20 \mathrm{~Hz}$ ASSR, hence this ASSR is not shown. Amplitudes are not shown either, as they yielded the same results as the SNRs. The dotted line indicates the significance cut-off, i.e., $4.8 \mathrm{~dB}$ SNR. Age groups (young, middle-aged, older) are displayed per row. The bars are clustered per hemisphere (LH, $\mathrm{RH}$ ) and represent the mean ASSR magnitude per hearing status, i.e., $\mathrm{NH}$ (dark fill) and $\mathrm{HI}$ (gray fill). Error bars indicate the $95 \%$ confidence interval. ${ }^{*} P \leq 0.05 ;{ }^{* *} P \leq 0.01$. 